\title{
Astrocytoma Following Scalp Radiotherapy in Infancy
}

\author{
Douglas W. Zochodne, J. Gregory Cairncross, Felix P. Arce, John C.F. MacDonald, \\ Warren T. Blume, John P. Girvin, and John C.E. Kaufmann
}

\begin{abstract}
A 24 year old woman with a 15 year history of intractable seizures underwent resection of a small low grade astrocytoma of the right mesial frontal lobe. The tumor arose beneath a benign scalp nevus that had been treated in infancy with radium patches and focal $\mathrm{x}$-irradiation. Neuropathological changes within the tumor were compatible with radiation injury. Meningiomas and sarcomas complicate cranial irradiation but the evidence that gliomas do so is less convincing. Our observations support an association between radiation exposure and the subsequent development of glial tumors.
\end{abstract}

RÉSUMÉ: Une patiente de 24 ans qui souffrait depuis 15 ans d'une épilepsie mal contrôlée a subi l'exérèse d'un petit astrocytome bien différencié. La tumeur a été retrouvée en frontal droit, au-dessous d'un naevus bénin du cuir chevelu traité durant l'enfance par des applications de radium et des rayons-X superficiels. La relation méningiome, sarcome et irradiation est bien démontrée dans la littérature. Nos observations semblent elles étayer une association gliome-irradiation, association pour laquelle la littérature est peu concluante.

Can. J. Neurol. Sci. $1984 ; 11: 475-478$

A variety of benign and malignant tumors develop in previously irradiated tissues. Sarcomas and meningiomas are now a recognized complication of cranial irradiation. Pertinent to the present case is the unexpectedly high incidence of intracranial meningiomas following low dose scalp radiotherapy for childhood tinea capitis (Beller et al., 1972; Modan et al., 1974; Munk et al., 1969; Spallone et al., 1979). Gliomas are reported in patients and experimental animals following radiation to the brain. The evidence supporting this association is not entirely convincing however. We report a patient who developed a right frontal astrocytoma beneath a scalp hemangioma irradiated in infancy. The unusual clinical and pathological findings in this case implicate radiation injury to the underlying brain in the pathogenesis of this brain tumor.

\section{CASE Report}

A 24-year-old right-handed woman with intractable seizures was admitted to the hospital for elective resection of a right mesial frontal contrast-enhancing lesion on computed tomographic (CT) scan. Her medical problems began in infancy. Two small cutaneous hemangiomas, one on the right anterior scalp and the other over the sacrum, were noted at birth. They measured $1.3 \times 1.2 \mathrm{~cm}$ and $2.0 \times 1.2 \mathrm{~cm}$, respectively. A series of radiation treatments for the skin lesions began ten weeks
Table 1: Prescribed treatment for the benign scalp hemangioma. A total skin dose of 1610 rads given by a combination of $x$-irradiation and radium patching over an 8 month period.

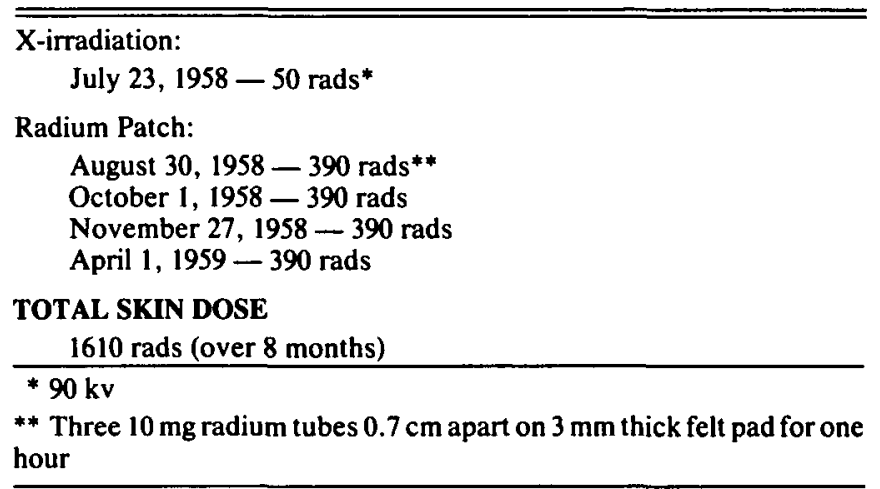

after birth. The right scalp hemangioma was treated by a combination of focal $x$-irradiation and radium patching (Table 1 ). The sacral lesion received seven focal $x$-irradiation treatments over a two year period. Both lesions eventually disappeared.

Staring spells began at age nine. At times the spells were associated with automatic behaviour, inappropriate laughter and adversive head and eye movements to the left. Repeated neurological examinations

From the Departments of Clinical Neurological Sciences, Pathology (Neuropathology) and Radiation Oncology, University of Western Ontario, London, Canada, and Ontario Cancer Treatment and Research Foundation

Received May 15, 1984. Accepted July 19, 1984.

Reprint requests to: J. Gregory Cairncross, M.D., 391 South Street, London, Ontario, Canada N6A 4G5 


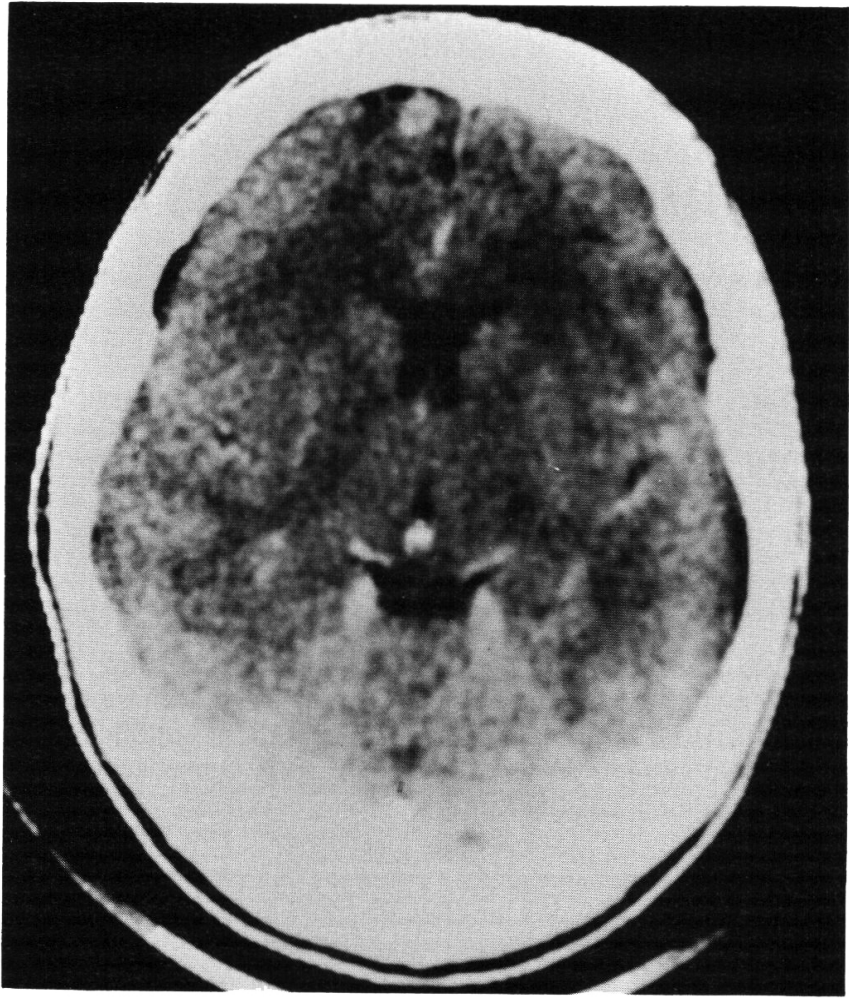

Figure I - A contrast enhanced CT scan demonstrating a right mesial frontal lesion.

were normal. EEG recordings on a number of occasions suggested a right hemisphere focus and pneumoencephalography disclosed a dilated right temporal horn. Cerebral angiography was normal. Various combinations of anticonvulsants failed to adequately control seizures over subsequent years. At age 23 a CT scan was performed for the first time. An unexpected area of low density with oval contrast enhancement was visualized in the right mesial frontal lobe (Figure 1). Surgical excision of the lesion was advised but declined. One year later the patient consented to a right frontal craniotomy. The general medical and neurological examinations again were normal. There was no family history of neurofibromatosis, tuberous sclerosis or other neurocutaneous disorders. At surgery the midportion of the superior frontal gyrus approximately $5 \mathrm{~cm}$ behind the frontal pole was discoloured, expanded, and hypovascular. A partial right parasagittal midfrontal resection including all of the superior frontal gyrus was performed. The postoperative course was uneventful and the patient was discharged from hospital ten days after surgery. There have been no seizures in the 2 years since surgery.

The pathological specimen measured $5.3 \times 4.0 \times 2.2 \mathrm{~cm}$. The superior frontal gyrus was widened, pearly-white in colour and of rubbery consistency. The cut surface revealed a distorted and irregularly thickened cortex (Figure 2a). The microscopic abnormalities were confined to the single widened gyrus. The cerebral cortex was replaced by a spindle cell neoplasm (Figure 2b). Individual tumor cells were characterized by elongated nuclei with fine dispersed chromatin and one or more small nucleoli, and eosinophilic ill-defined fibrillary cytoplasm and processes. The cells were arranged in a network of bundles with palisading in some areas. The tumor cells were identified as astrocytic by the presence of phosphotungstic acid hematoxylin-stained (PTAH) fibrils and glial fibrillary acid protein (immunoperoxidase). Tumor cells extended to the pial surface. Deep to the cellular part of the tumor was a large cystic area occupying most of the involved gyrus. In this cystic region were large numbers of hyalinized, occluded blood vessels some with calcified walls (Figure 2c). Small numbers of tumor cells separated the vessels. Hyalinized vessels were also present in the overlying cellular part of the tumor. The underlying white matter contained a few isolated ectopic neurons but was otherwise normal. Laterally the tumor merged with uninvolved cortex. The margins of the resection were free of tumor.
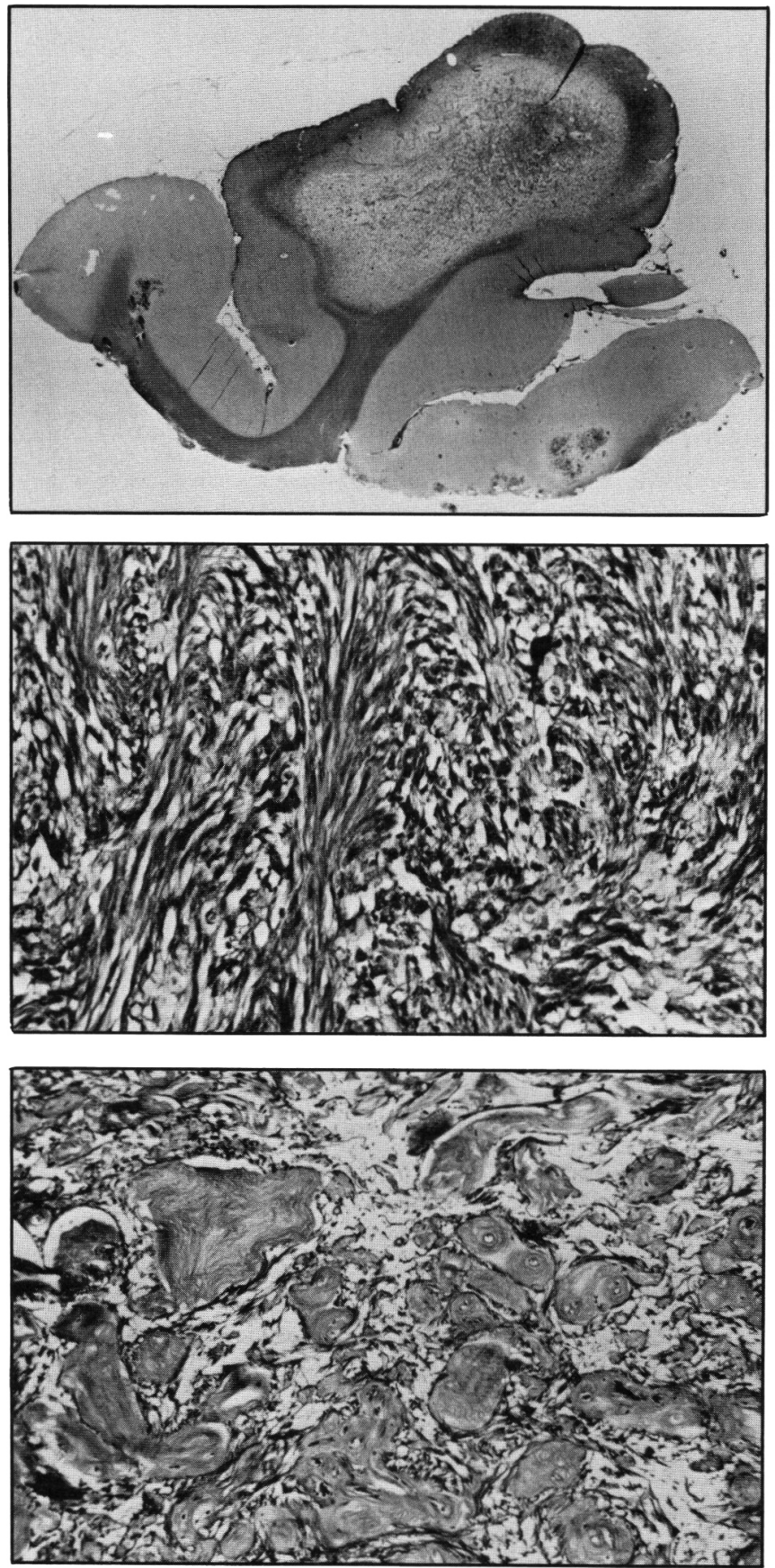

Figure 2 - Photomicrographs of a) the surgical specimen showing tumor and cystic change in the superior frontal gyrus $(P T A H, 2.3 X)$, b) the tumor composed of bundles of spindle cells with elongated fibrillary cytoplasm (PTAH, 140X), and c) numerous blood vessels with fibrillary hyalin walls (some occluded) and interposed tumor cells (PTAH, 56X).

The morphology and tinctorial properties of the tumor cells, degree of cellularity, and absence of mitoses, necrosis, or hyperplastic vessels pointed to an unusual slow growing spindle cell astrocytoma of Kernohan, grade II.

\section{Discussion}

The best experimental evidence that glial tumors follow cranial irradiation comes from the work of Haymaker et al. (1972) 

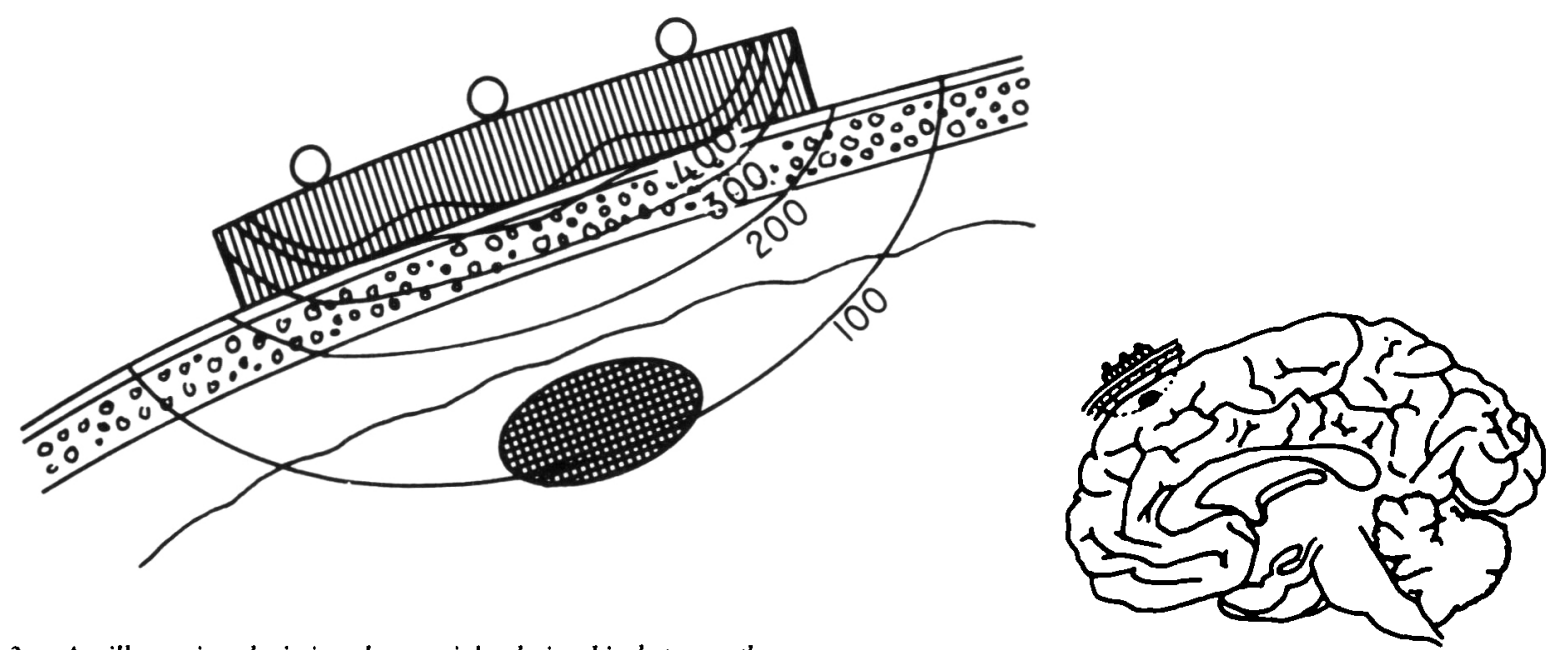

Figure 3-An illustration depicting the spacial relationship between the radium applicator and the underlying superior frontal gyrus. Computed dose-rate curves from three $10 \mathrm{mg}$, radium sources are superimposed.

in Macaca mulatta monkeys. Despite an exceedingly low incidence of spontaneous brain tumors in this species, gliomas developed in 3 of 32 monkeys following whole body proton beam irradiation (600-800 rads). Vascular changes typical of radiation injury were found adjacent to tumor in two of the cases. There are a number of reports of gliomas in patients following cranial irradiation (Albert et al., 1966; Bachman and Ostrow, 1978; Barnes et al., 1982; Chung et al., 1981; Clifton et al., 1980; Cohen et al., 1981; Jones, 1960; Kleriga et al., 1978; Komaki et al., 1977; Pearl et al., 1980; Piatt et al., 1983; Preissig et al., 1979; Robinson, 1978; Saenger et al., 1960; Sogg et al., 1978; Steinbok, 1980). They have been summarized in table form by Piatt et al. (1983). There are potential criticisms of many of these cases such that the link between radiation exposure and glioma induction is substantially weakened. Detailed neuropathological descriptions of the tumor and surrounding tissue are absent in some instances (Albert et al., 1966; Jones, 1960; Saenger et al., 1960). Since reactive glial proliferation may occur in the irradiated brain and be mistaken for tumor, a precise pathological diagnosis is essential. It is not clear in all reports that inherited conditions which predispose to gliomas have been considered and excluded (Albert et al., 1966; Bachman and Ostrow, 1978; Barnes et al., 1982; Clifton et al., 1980; Jones, 1960; Kleriga et al., 1978; Pearl et al., 1980; Piatt et al., 1983; Robinson, 1978; Saenger et al., 1960; Sogg et al., 1978; Steinbok, 1980). In some cases the radiation field and its relationship to the location of the tumor are not discussed (Albert et al., 1966; Jones, 1960; Saenger et al., 1980), or gliomas have developed following radiotherapy to the whole brain (Bachman and Ostrow, 1978; Barnes et al., 1982; Chung et al., 1981; Cohen et al., 1981; Kleriga et al., 1978; Pearl et al., 1980). Those instances in which glial tumors arise within a limited CNS radiation field are more persuasive (Clifton et al., 1980; Komaki et al., 1977; Piatt et al., 1983; Preissig et al., 1979; Robinson, 1978; Sogg et al., 1978; Steinbok, 1980). Lastly, many radiation associated gliomas have developed in patients in whom radiotherapy was prescribed for a pre-existing neoplastic condition (Bachman and Ostrow, 1978; Barnes et al., 1982; Chung et al., 1981; Clifton et al., 1980; Cohen et al., 1981; Jones, 1960; Kleriga et al., 1978; Komaki et al., 1977; Pearl et al., 1980; Piatt et al., 1983; Preissig et al., 1983; Robinson, 1978; Sogg et al., 1978). This raises the possibility that the gliomas were not radiation-induced but rather developed as a consequence of an underlying "tumor diathesis".

The striking features of the present case include the development of a glioma in the cerebral cortex beneath a small radiation field and the unusual pathological findings of a spindle cell astrocytoma with vascular changes indicative of radiation injury. From the radiation records the doses to skin and underlying tissues have been estimated and illustrated in the form of isodose curves (Figure 3). These approximations indicate that the superficial layers of underlying brain were exposed to very little radiation ( $<600$ rads). We cannot be absolutely certain that the diseased gyrus was immediately beneath the scalp lesion and radium patches during the period of radiation exposure. The scalp lesion was not visible at the time of surgery and therefore its precise relationship to the underlying tumor could not be ascertained. Had the hemangioma persisted, relative changes in position between the scalp and underlying brain due to growth may not have resolved this issue. The whorled, spindle cell appearance of the tumor is unusual but the glial filament positive staining is evidence for an astrocytic origin and against being sarcomatons or mesenchymal. Glial filament staining does not distinguish between neoplastic and reactive astrocytes, but the expanded and densely cellular nature of the lesion is indicative of tumor rather than of reactive gliosis. Numerous hyalinized, occluded blood vessels with calcified walls and cystic cavitation are found within the tumor, findings characteristic of radiation injury (Rottenberg et al., 1977). This observation supports the radiation-associated nature of this glioma and furthermore suggests an intimate relationship between the scalp hemangioma and superior frontal gyrus during the period of exposure to radiation.

The majority of radiation-associated gliomas are highly anaplastic tumors developing in heavily irradiated brain. In the present case the tumor was histologically benign and followed low dose exposure. Conceivably there is a relationship between the radiation absorbed dose and the tumor histology, although this must be regarded as highly speculative. The tumor was confined to the superficial layers of the cerebral cortex. Most 
gliomas are considerably larger at the time of diagnosis and are found in the subcortical white matter. Perhaps the intractable seizures led to early diagnosis and treatment at a time when the effects of radiation on the underlying brain were not obscured by a large tumor mass. There is insufficient information available to comment on the relative susceptibilities of the infant and adult brain to the oncogenic effects of ionizing radiation.

However compelling the circumstantial evidence in this case, there is no direct proof of a cause and effect relationship between brain irradiation and glioma induction. With this proviso, the present case would appear to strengthen the association between radiation exposure and the development of glial tumors. This case once again calls to our attention the potential hazards of radiation as a treatment for benign conditions of any kind, especially in young patients.

\section{REFERENCES}

Albert RE, Omran AR, Brauer EW, et al (1966) Follow-up study of patients treated by $\mathrm{X}$-ray for tinea capitis. Am J Public Health 56: 2114-2120.

Bachman DS, Ostrow PT (1978) Fatal long-term sequela following radiation "cure" for ependymoma. Ann Neurol 4: 319-321.

Barnes AE, Liwnicz BH, Schellhas HF, Altshuler G, Aron BS, Lippert WA (1982) Successful treatment of placental choriocarcinoma metastatic to brain followed by primary brain glioblastoma. Gynecol Oncol 13: 108-114.

Beller AJ, Feinsod M, Sahar A (1972) The possible relationship between small dose irradiation to the scalp and intracranial meningomas. Neurochirurgia 4: 135-143.

Chung CK, Stryker JA, Cruse R, Vannuci R, Towflight J (1981) Glioblastoma multiforme following prophylactic cranial irradiation and intrathecal methotrexate in a child with acute lymphocytic leukemia. Cancer 47: 2563-2566.

Clifton MD, Amromin GD, Perry MC, Abadir R, Watts C, Levy N (1980) Spinal cord glioma following irradiation for Hodgkin's disease. Cancer 45: 2051-2055.
Cohen MS, Kushner MJ, Dell S (1981) Frontal lobe astrocytoma following radiotherapy for medulloblastoma. Neurol (NY) 31: 616-619.

Haymaker W, Rubinstein LJ, Miquel J (1972) Brain tumors in irradiated monkeys. Acta Neuropath 20: 267-277.

Jones A (1960) Supervoltage x-ray therapy for intracranial tumors. Ann R Coll Surg Engl 27: 310-354.

Kleriga E, Sher JH, Nallainathan S, Stein SC, Sacher M (1978) Development of cerebellar malignant astrocytoma at site of medulloblastoma treated 11 years earlier. J Neurosurg 49: 445-449.

Komaki S, Komaki R, Choi H, Correa-Paz F (1977) Radiation and drug induced intracranial neoplasm with angiographic demonstration. Neurol Med Chir (Tokyo) 17: 55-62.

Modan B, Baidatz D, Mart H, Steinitz R, Levin SG (1974) Radiationinduced head and neck tumors. Lancet 1: 277-279.

Munk J, Peyser E, Gruszkiewicz J (1969) Radiation-induced intracranial meningiomas. Clin Radiol 20: 90-94.

Pearl GS, Mirra SS, Miles ML (1980) Glioblastoma multiforme occurring 13 years after treatment of a medulloblastoma. Neurosurgery 6: 546-551.

Piatt JH, Blue JM, Schold SC, Burger PC (1983) Glioblastoma multiforme after radiotherapy for acromegaly. Neurosurgery 13: 85-89.

Preissig SH, Bohmfalk GL, Reichel GW, Smith MT (1979) Anaplastic astrocytoma following radiation for a glomus jugulare tumor. Cancer 43: 2243-2247.

Robinson RG (1978) A second brain tumor and irradiation. J Neurol Neurosurg Psychiat 41: 1005-1012.

Rottenberg DA, Chernik NL, Deck MDF, Ellis F, Posner JB (1977) Cerebral necrosis following radiotherapy of extracranial neoplasms. Ann Neurol 1: 339-357.

Saenger EL, Silverman FN, Sterling TD, Turner ME (1960) Neoplasia following therapeutic irradiation for benign conditions in childhood. Radiology 74: 889-904.

Sogg RL, Donaldson SS, Yorke CH (1978) Malignant astrocytoma following radiotherapy of a craniopharyngioma. J Neurosurg 48: 622-627.

Spallone A, Gagliardi FM, Vagnozzi R (1979) Intracranial meningiomas related to external cranial irradiation. Surg Neurol 12: 153-159.

Steinbok P (1980) Spinal cord glioma after multiple fluoroscopies during artificial pneumothorax treatment of pulmonary tuberculosis. $J$ Neurosurg 52: 838-841. 